\title{
Fluid oscillations in a laboratory geyser with a bubble trap
}

\author{
Maxwell L. Rudolph ${ }^{\mathrm{a}, *}$, Robert A. Sohn ${ }^{\mathrm{b}}$, Einat $\mathrm{Lev}^{\mathrm{c}}$ \\ ${ }^{a}$ Department of Earth and Planetary Sciences, University of California, Davis, CA USA \\ ${ }^{b}$ Woods Hole Oceanographic Institution, Woods Hole, MA, USA \\ ${ }^{c}$ Lamont-Doherty Earth Observatory, Palisades, NY, USA
}

\begin{abstract}
Geysers are rare geologic features that episodically erupt water and steam. While it is understood that the eruptions are triggered by the conversion of thermal to kinetic energy during decompression of hot fluids, geysers commonly exhibit a range of dynamic behaviors in-between and during eruptions that have yet to be adequately explained. In-situ measurements of temperature and pressure as well as remote geophysical techniques have revealed oscillatory behavior across a range of timescales, ranging from eruption cycles to impulsive bubble collapse events. Many geysers, including Old faithful in Yellowstone National Park, USA, are believed to have offset subsurface reservoirs (referred to as a 'bubble trap') that can trap and accumulate noncondensable gas or steam entering the system. The impact of a bubble trap on the dynamic behaviors of the system, however, has not been fully established. We constructed a laboratory bubble trap and performed a series of experiments to study how fluids oscillate back and forth between the eruption conduit and laterally-offseet reservoir in-between eruptions. We present a new theoretical model based on Hamiltonian mechanics that successfully predicts the oscillation frequencies observed in our experiments based on the conduit system geometry, the amount of gas that has accumulated in the bubble trap, and the amount of liquid water in the system. We demonstrate that when scaled to Old Faithful Geyser, this mechanism is capable of producing oscillations at the observed frequencies.
\end{abstract}

Keywords: geysers, oscillations, bubble trap, tremor

\footnotetext{
${ }^{*}$ Corresponding author

Email address: maxrudolph@ucdavis.edu (Maxwell L. Rudolph)
} 


\section{Introduction}

The behavior of geysers has been a topic of research for several centuries (e.g. Bunsen, 1847, Mackenzie, 1811). Geysers are rare relative to hot springs or fumaroles, which continuously discharge hot water or steam, which has lead to the suggestion that a special subsurface geometry or combination of transport

5 properties is required to give rise to episodic eruptions. Many geyser models conceptualize the conduit §ystem as consisting of one or more vertical pipes/chambers (e.g. Steinberg and Merzhanov, 1981; Dowden et al., 1991; Ingebritsen and Rojstaczer, 1996; Kagami, 2012; Anatolyevich, 2013; O'Hara and Esawi, 2013. Namiki et al., 2014, Munoz-Saez et al., 2015b; Alexandrov et al., 2016). However, recent in situ and remote geophysical investigations of geysers have renewed interest in the old idea that the eruption conduit of many geysers appears to be connected to a laterally offset cavity (Figure 1A), or a 'bubble trap' (e.g. Cros et al. 2011; Vandemeulebrouck et al., 2013, 2014, Belousov et al., 2013, Rudolph and Sohn, 2017). The dynamics of a geyser with a bubble trap (Figure 1A) differ significantly from that of vertical-pipe models because any non-condensible gas or vapor that ascends into the conduit system is sequestered in the bubble trap. As a consequence, the fluid in the conduit loads and interacts with the highly compressible trapped steam/gas. When this system is perturbed (e.g., by fluid input), it can respond by oscillating at resonant frequencies determined by the conduit geometry and the amount of fluid in the system (Rudolph and Sohn, 2017). Previous laboratory experiments involving a closed system analogue geyser demonstrated that the presence of a bubble trap within the conduit can lead to differences in eruption behavior associated with flushing of the shallow part of the conduit vs. the entire conduit (Adelstein et al. 2014).

While a great deal of research has been directed towards understanding the fluid mechanics and thermodynamics of geyser eruptions, it has become clear that geysers also display interesting dynamical behaviors in-between eruptions, during the recharge phase (e.g. Birch and Kennedy, 1972, Hutchinson et al., 1997, Kedar et al., 1998, Karlstrom et al., 2013, Vandemeulebrouck et al., 2014, Munoz-Saez et al., 2015b). In particular, the liquid level/pressure in the eruption conduit oscillates at characteristic periods, ranging from seconds (Figure 2) (Birch and Kennedy, 1972, Hutchinson et al., 1997; Munoz-Saez et al., 2015b) to tens of seconds (Karlstrom et al., 2013), and the oscillation period can systematically change as the system regains mass and energy leading up to an eruption (Karlstrom et al. 2013; Rudolph and Sohn, 2017). The observation that individual geysers exhibit pressure oscillations at characteristic frequencies suggests that a geyser's subsurface conduit geometry controls the resonant oscillation frequency, and the observation that the resonant frequency can change as mass and energy are added to the system demonstrates that time evolution of fluid mass and thermodynamic state could affect the resonant frequency. These observations motivate the development of a theory relating the resonant frequency of geyser pressure oscillations to the subsurface conduit geometry and fluid mass as a way to constrain these important, but hard-to-observe parameters.

In order to study internal fluid oscillations in bubble trap geyser configurations and to test the theoretical 
model of (Rudolph and Sohn, 2017), we built a laboratory analog geyser with a bubble trap geometry and ran a series of perturbation experiments monitoring the gas pressure in the bubble trap, the fluid pressure at the bottom of the conduit, and the liquid level in the conduit. These experiments produced pressure timeseries that are analogous to pressure records measured within the conduits of natural geysers in-between eruptions (Hutchinson et al., 1997; Kedar et al., 1998; Munoz-Saez et al., 2015a), and that can be used to test theoretical predictions for fluid motion. Our observations demonstrate that the theoretical model of Rudolph and Sohn (2017) does not agree with the frequency of the oscillations in our laboratory geyser. We develop a new equation of motion (EOM) for fluid motion in a bubble trap conduit geometry based on Hamiltonian mechanics (starting from a statement of conservation of energy, rather than balance of forces). Through linearization of the EOM, we obtain an analytical expression relating the resonant frequency of the system to the model parameters. We find that the new equation of motion and the expression for resonant frequency are in good agreement with the laboratory experiments, and that resonant frequency depends primarily on the total fluid length of the system as well as the amount of gas present in the bubble trap. Our results demonstrate that the new theory may be used to constrain the subsurface conduit geometry and fluid mass, and possibly the thermodynamic state, of natural systems if the frequency of the conduit pressure oscillations is measured. 

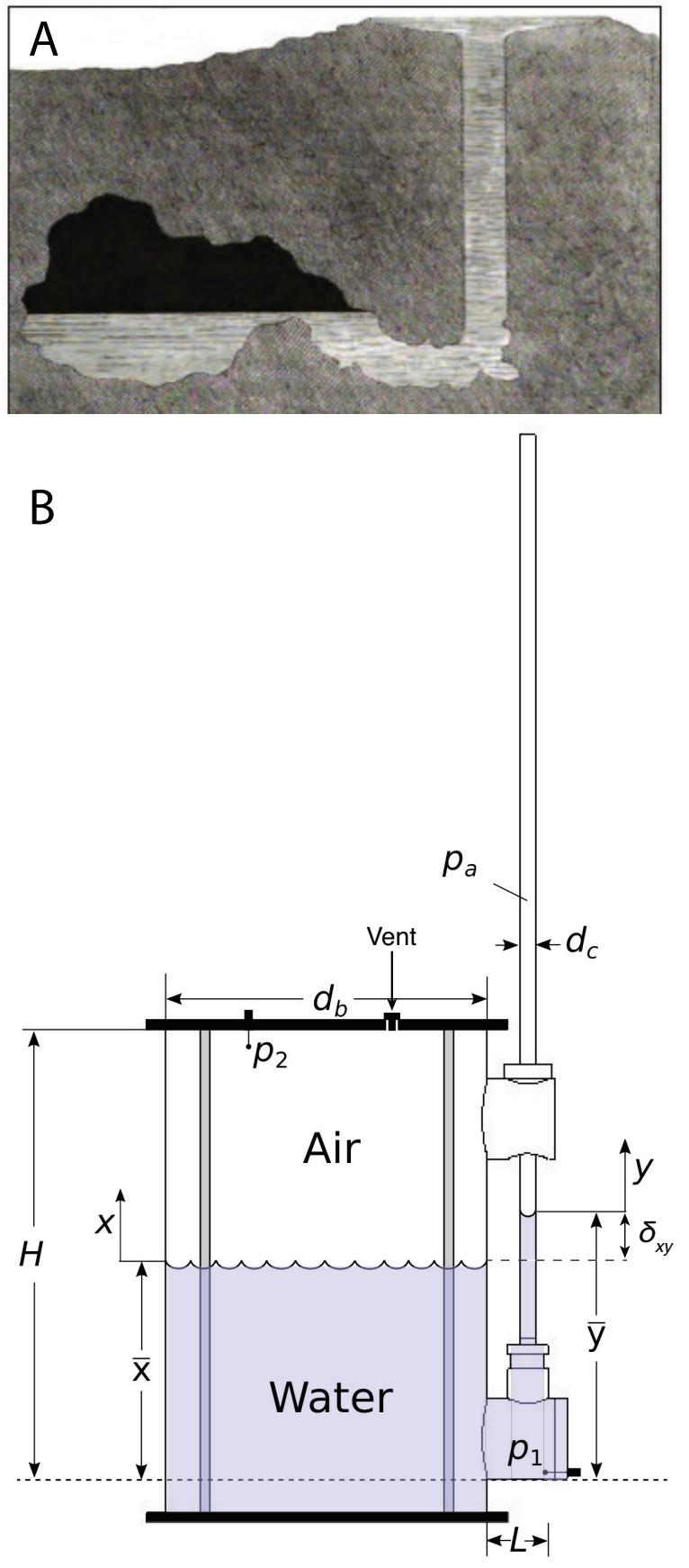

Figure 1: (A) A schematic cross-section of a geyser system with an offset 'bubble trap' (Mackenzie 1811). (B) Schematic diagram of the experimental setup, including definitions of the geometric parameters. Dimensions of the analog geyser are provided in Table 1 

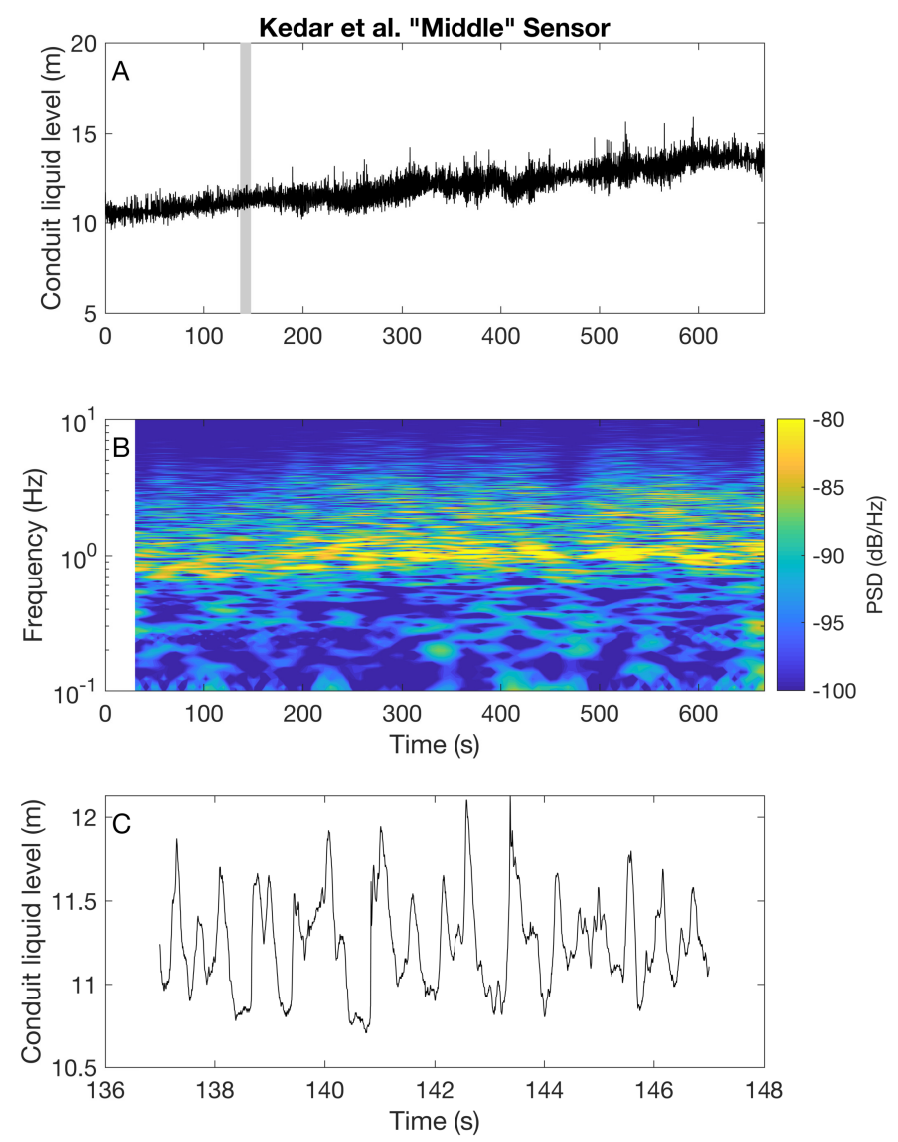

Figure 2: (A) Pressure signals recorded within the eruption conduit of Old Faithful Geyser during the recharge phase between eruptions by Kedar et al. (1996) using their 'middle' pressure transducer. (B) A spectrogram of the signal reveals a peak in power around $1 \mathrm{~Hz}$. The color scale indicates power spectral density (PSD). (C) The shape of pressure oscillations during the period idicated with the gray band in (A). Modified from Rudolph and Sohn (2017). 


\section{Laboratory geyser experiments}

\subsection{Experimental setup}

We constructed a laboratory geyser to test the prediction for internal fluid oscillation frequency from Rudolph and Sohn (2017). The laboratory geyser (Figure 1) contains three primary components: 1) a large

55 likely the case in many natural systems.

\begin{tabular}{|c|c|c|}
\hline Symbol & Name & Value (if constant) \\
\hline$H$ & Bubble trap height & $37.15 \mathrm{~cm}$ \\
$\bar{x}$ & Equilibrium liquid level in bubble trap & - \\
$\bar{y}$ & Equilibrium liquid level in conduit & - \\
$d_{b}$ & Bubble trap inner diameter & 11 inches $(27.94 \mathrm{~cm})$ \\
$S_{b}$ & Bubble trap area & $613 \mathrm{~cm}^{2}$ \\
$d_{c}$ & Conduit inner diameter & $3 / 8$ inch $(0.953 \mathrm{~cm})$ or 1 inch $(2.54 \mathrm{~cm})$ \\
$S_{c}$ & Conduit area & $0.713 \mathrm{~cm}^{2}$ or $5.07 \mathrm{~cm}^{2}$ \\
$L$ & Lateral connector length & $7.37 \mathrm{~cm}^{2}$ \\
$S_{l}$ & Lateral connector area & $7.92 \mathrm{~cm}^{2}$ \\
\hline
\end{tabular}

Table 1: Geometric parameters for the laboratory geyser. reservoir containing a gas layer overlying a liquid (i.e., bubble trap), 2) a lateral connector, and 3) a tall,
narrow vertical conduit open to the atmosphere. The reservoir and conduits are made of acrylic plastic,
and the reservoir lids and mechanical fasteners are made of Delrin plastic. Metal rods were used to stiffen
the reservoir assembly and provide an airtight seal between the lid and bubble trap walls. Conduits of two
different internal diameters ( 1 inch $(2.54 \mathrm{~cm})$ and $3 / 8$ inch $(0.95 \mathrm{~cm}))$ were constructed to assess the impact
of conduit size on the system dynamics. The conduit diameters were chosen to be large enough to mitigate
the effects of surface tension, and the reservoir diameter was chosen to be large relative to the conduit as is reservoir containing a gas layer overlying a liquid (i.e., bubble trap), 2) a lateral connector, and 3) a tall,
narrow vertical conduit open to the atmosphere. The reservoir and conduits are made of acrylic plastic,
and the reservoir lids and mechanical fasteners are made of Delrin plastic. Metal rods were used to stiffen
the reservoir assembly and provide an airtight seal between the lid and bubble trap walls. Conduits of two
different internal diameters ( 1 inch $(2.54 \mathrm{~cm})$ and $3 / 8$ inch $(0.95 \mathrm{~cm}))$ were constructed to assess the impact
of conduit size on the system dynamics. The conduit diameters were chosen to be large enough to mitigate
the effects of surface tension, and the reservoir diameter was chosen to be large relative to the conduit as is reservoir containing a gas layer overlying a liquid (i.e., bubble trap), 2) a lateral connector, and 3) a tall,
narrow vertical conduit open to the atmosphere. The reservoir and conduits are made of acrylic plastic,
and the reservoir lids and mechanical fasteners are made of Delrin plastic. Metal rods were used to stiffen
the reservoir assembly and provide an airtight seal between the lid and bubble trap walls. Conduits of two
different internal diameters ( 1 inch $(2.54 \mathrm{~cm})$ and $3 / 8$ inch $(0.95 \mathrm{~cm}))$ were constructed to assess the impact
of conduit size on the system dynamics. The conduit diameters were chosen to be large enough to mitigate
the effects of surface tension, and the reservoir diameter was chosen to be large relative to the conduit as is reservoir containing a gas layer overlying a liquid (i.e., bubble trap), 2) a lateral connector, and 3) a tall,
narrow vertical conduit open to the atmosphere. The reservoir and conduits are made of acrylic plastic,
and the reservoir lids and mechanical fasteners are made of Delrin plastic. Metal rods were used to stiffen
the reservoir assembly and provide an airtight seal between the lid and bubble trap walls. Conduits of two
different internal diameters ( 1 inch $(2.54 \mathrm{~cm})$ and $3 / 8$ inch $(0.95 \mathrm{~cm}))$ were constructed to assess the impact
of conduit size on the system dynamics. The conduit diameters were chosen to be large enough to mitigate
the effects of surface tension, and the reservoir diameter was chosen to be large relative to the conduit as is reservoir containing a gas layer overlying a liquid (i.e., bubble trap), 2) a lateral connector, and 3) a tall,
narrow vertical conduit open to the atmosphere. The reservoir and conduits are made of acrylic plastic,
and the reservoir lids and mechanical fasteners are made of Delrin plastic. Metal rods were used to stiffen
the reservoir assembly and provide an airtight seal between the lid and bubble trap walls. Conduits of two
different internal diameters ( 1 inch $(2.54 \mathrm{~cm})$ and $3 / 8$ inch $(0.95 \mathrm{~cm}))$ were constructed to assess the impact
of conduit size on the system dynamics. The conduit diameters were chosen to be large enough to mitigate
the effects of surface tension, and the reservoir diameter was chosen to be large relative to the conduit as is reservoir containing a gas layer overlying a liquid (i.e., bubble trap), 2) a lateral connector, and 3) a tall,
narrow vertical conduit open to the atmosphere. The reservoir and conduits are made of acrylic plastic,
and the reservoir lids and mechanical fasteners are made of Delrin plastic. Metal rods were used to stiffen
the reservoir assembly and provide an airtight seal between the lid and bubble trap walls. Conduits of two
different internal diameters ( 1 inch $(2.54 \mathrm{~cm})$ and $3 / 8$ inch $(0.95 \mathrm{~cm}))$ were constructed to assess the impact
of conduit size on the system dynamics. The conduit diameters were chosen to be large enough to mitigate
the effects of surface tension, and the reservoir diameter was chosen to be large relative to the conduit as is

$$
\text { . }
$$
\begin{tabular}{|c|c|c|}
\hline Symbol & Name & Value (if constant) \\
\hline$H$ & Bubble trap height & $37.15 \mathrm{~cm}$ \\
$\bar{x}$ & Equilibrium liquid level in bubble trap & - \\
$\bar{y}$ & Equilibrium liquid level in conduit & - \\
$d_{b}$ & Bubble trap inner diameter & 11 inches $(27.94 \mathrm{~cm})$ \\
$S_{b}$ & Bubble trap area & $613 \mathrm{~cm}^{2}$ \\
$d_{c}$ & Conduit inner diameter & $3 / 8$ inch $(0.953 \mathrm{~cm})$ or 1 inch $(2.54 \mathrm{~cm})$ \\
$S_{c}$ & Conduit area & $0.713 \mathrm{~cm}^{2}$ or $5.07 \mathrm{~cm}^{2}$ \\
$L$ & Lateral connector length & $7.37 \mathrm{~cm}^{2}$ \\
$S_{l}$ & Lateral connector area & $7.92 \mathrm{~cm}^{2}$ \\
\hline
\end{tabular}

\subsection{Monitoring and measurement techniques}

Two pressure transducers (Druck model MXR 2.5 PSIG differential pressure bridge transducers) were

65 upper lid of the reservoir. The first measures fluid pressure at the bottom of the conduit while the second measures gas pressure in the reservoir. A third pressure sensor, mounted in the data logger, measured atmospheric pressure. Each transducer is conditioned by an instrumentation amplifier with a gain of 251 (Analog Devices INA118, $\mathrm{Rg}=200 \Omega$ ). The signal from the three pressure transducers was digitized at 40

$\mathrm{Hz}$ by an oversampled dual channel 12 bit analog-to-digital converter. Nominally the weight of 1 bit with

Table 1: Geometric parameters for the laboratory geyser. 
gain equals $4.86 \mu \mathrm{V}$ and full range spans 0 to $19.9 \mathrm{mV}$. The transducer sensitivity was $1.45 \mu \mathrm{V} / \mathrm{Pa}$. The full scale differential pressure limit as function of gain and reference used was $13.1 \times 10^{3} \mathrm{~Pa}$. A real time clock (RTC) provided a time/date stamp at the beginning and end of every run, and the $40 \mathrm{~Hz}$ sampling rate was validated with a tachometer. The water temperature in the reservoir was also measured with a DS18B20 solid state sensor before and after each experiment with a resolution of $0.1{ }^{\circ} \mathrm{C}$.

The experiments were filmed using a Canon VIXIA R60 video camera, with a sensor resolution of 1920 x 1080 pixels and a frame rate of 29.97 frames per second. The camera was oriented perpendicular to the conduit and focused on the top of the liquid column in the conduit (Figure $3 \mathrm{~A}$ ).

\subsection{Experimental procedure}

To conduct the experiments, the apparatus was filled with room temperature water to specified levels, and the equilibrium parameters at atmospheric pressure were recorded $\left(\bar{x}, \bar{y}, p_{1}, p_{2}, p_{\text {atm }}\right)$. For each experimental run, the liquid level in the conduit was perturbed (by compression or decompression of the overlying gas) and the system response was recorded, generating a set of time-series data for each experimental run. A sample of the oscillatory signals recorded during one run are shown in Figure 3

In the first set of experiments, the reservoir lid was vented (Figure 1) to the atmosphere so that the gas pressure above the liquids in both the conduit and bubble trap remained constant at atmospheric during the fluid oscillations. These 'open system' experiments provide a useful means to study the simplest possible kinds of oscillations that do not involve gas compression. A summary of the resonant frequencies observed during all of the open system runs is shown in Figure 4. Because the bubble trap is vented to the atmosphere in the open system runs, the pressure sensor in the bubble trap $\left(p_{2}\right)$ records only the atmospheric pressure and does not provide useful information regarding the fluid motion, but the pressure sensor at the bottom of the conduit $\left(p_{1}\right)$ measured oscillatory pressure signals associated with dynamic pressure and the video camera measure changes in the conduit liquid level.

A second set of experiments was conducted with the reservoir lid sealed, creating a bubble trap above the liquid in the reservoir such that the trapped air above the liquid must compress and expand as the liquid level changes. A typical sequence of runs involved closing the reservoir lid after a specified amount of liquid had been added to the system, such that the equilibrium gas pressure in the reservoir was atmospheric and the liquid levels in the reservoir and conduit were equal. The system response to a perturbation was recorded, and, with the reservoir sealed, water was added to the system through the top of the conduit. This had the effect of compressing the gas in the bubble trap, and the amount of compression (i.e., the extra load on the gas above atmospheric pressure) was controlled by the difference in the liquid levels between the bubble trap and conduit $\left(\delta_{x y}\right)$. Water was added until a specified $\delta_{x y}$ value was reached, and the new equilibrium value of $x_{0}$ was noted. The system response to a perturbation was recorded, and this process was repeated for increasing values of $\delta_{x y}$ until the conduit was too full to continue adding water. 


\section{Data Analysis}

For each experimental run, we manually extracted the data region of interest containing the response to the perturbation, and estimated the resonant frequency by fitting to the signal a function of the form $x(t)=e^{-t / c_{1}} \times\left(c_{2} \cos \left(2 \pi c_{3} t\right)+c_{4} \sin \left(2 \pi c_{3} t\right)\right)+c_{5}$ where $c_{1}-c_{5}$ are the fitting parameters, and $t$ is time. This function represents the solution to a damped harmonic oscillator. The parameter $c_{3}$ is the

oscillation frequency, related to the angular frequency $\omega$ through $\omega=2 \pi c_{3}$. Parameter estimates and confidence intervals were generated using the fit function in MATLAB R2016b, using the non-linear least squares method and the 'Bisquare Robust' option. We also estimated the signal-to-noise ratio of each perturbation experiment based on the variance of the oscillation signal relative to that of background noise levels measured when the system was at rest. We show an example of a pressure time series along with the results of the curve fitting procedure in Figure 3 .

We analyzed the video files in MATLAB to detect the vertical position of the liquid free surface in the conduit and generate time-series data of this parameter (Figure $3 \mathrm{~A}, \mathrm{~B}$ ). The resonant frequency of each perturbation experiment was estimated using the same procedure as described above for the pressure signals (Figure $3 \mathrm{C}$ ). The uncertainty in position of the free-surface is set by the pixel size, which is much smaller than the amplitude of the movement of the liquid ( $>50$ pixels) in at least the first five oscillations of each run, and does not impact the calculation of the dominant frequency. 

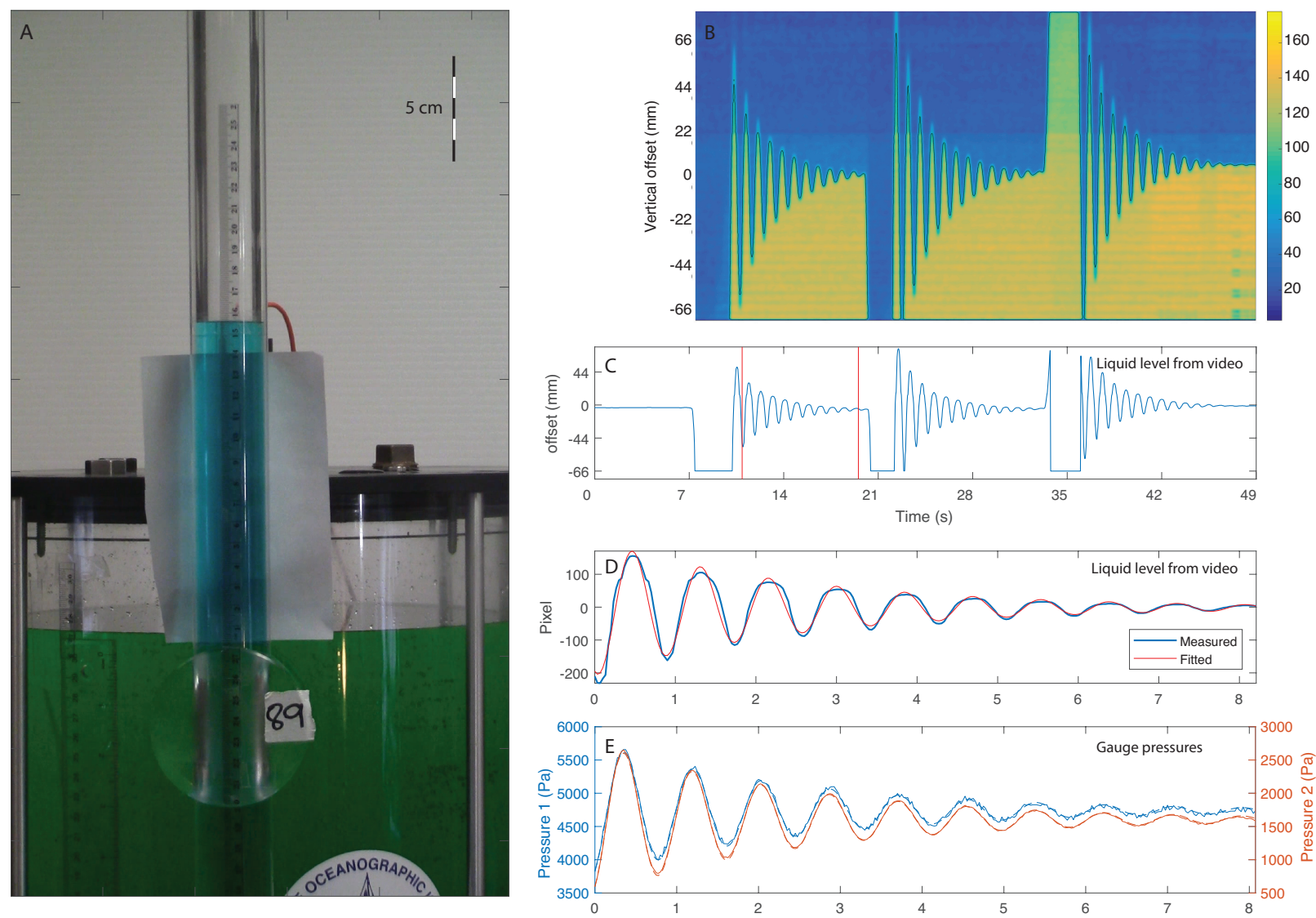

Figure 3: Example of the analysis performed on videos of the experiments. (A) A frame from the video of one of the closedsystem experiments. (B) The magnitude of the red image channel along the vertical cross-section over time. The black contour identifies the position of the top of the liquid in the conduit. (C) A time series showing the vertical position of the conduit fluid surface over time during three repeated runs of the same experimental configuration. Red lines mark the intervals used for curve fitting. (D) Measurements from video (blue) and fitted curve (red), using a damped oscillator equation. (E) Gauge pressure (solid lines) measured at $P_{1}$ (left axis) and $P_{2}$ (right axis). Dashed lines show a damped oscillator fit.

\section{Mathematical Model}

In developing a mechanical model for the laboratory geyser, we use similar simplifying assumptions to Rudolph and Sohn (2017). We treat the liquid water in the conduit, bubble trap, and lateral connector as an incompressible fluid. We treat the air in the bubble trap as a diatomic Ideal Gas. During oscillations, we assume that the gas undergoes adiabatic compression/decompression. We develop an equation of motion for the laboratory geyser starting from the principle of energy conservation (neglecting viscous dissipation). The total energy $E=T+U$ is the sum of the liquid kinetic energy $T$ and the potential energy $U$, which includes contributions from both the gravitational potential energy of the liquid and the internal energy of the trapped gas. Figure 1 shows a schematic of the laboratory geyser along with the definitions of key mathematical symbols, with values given in Table 1 and a complete list of mathematical symbols in Table 2 
We use $\bar{x}$ and $\bar{y}$ to represent the equilibrium water levels in the bubble trap and conduit. The perturbations to the liquid levels are $x$ and $y$, and we denote time derivatives using $\dot{x} \equiv \frac{d x}{d t}$. We begin by writing the kinetic energy,

$$
T=\frac{1}{2} \rho S_{b}(\bar{x}+x) \dot{x}^{2}+\frac{1}{2} \rho S_{c}(\bar{y}+y) \dot{y}^{2}+\frac{1}{2} \rho S_{l} L\left(-\frac{S_{b}}{S_{l}} \dot{x}\right)^{2},
$$

where the three terms represent the contributions due to fluid motion in the bubble trap, conduit, and lateral connector, respectively. The term $-\frac{S_{b}}{S_{l}} \dot{x}$ is the velocity of liquid in the lateral connector, determined from conservation of fluid mass. Next, we write an expression for the potential energy, where the first term corresponds to gravitational potential energy and the second corresponds to the internal energy of the compressed ideal gas in the bubble trap and in the atmosphere buffering the open conduit,

$$
U=\frac{\rho g}{2}\left[S_{b}(\bar{x}+x)^{2}+S_{c}(\bar{y}+y)^{2}\right]+\left(\alpha P_{b} V_{b}+\alpha P_{a} V_{a}\right)
$$

where $\alpha=5 / 2$ for a diatomic ideal gas, and $P_{b}, V_{b}$ are the gas pressure and volume in the bubble trap and $P_{a}$ and $V_{a}$ are the atmospheric pressure and volume (addressed below). The factor of $1 / 2$ in the first term which describes gravitational potential energy arises because the center of mass of the fluid in the conduit or bubble trap is displaced only half as far as the gas/liquid interface. Assuming adiabatic compression, the pressure and volume vary according to $P / P_{0}=\left(V / V_{0}\right)^{-\gamma}$ where the subscript 0 refers to the equilibrium state and $\gamma$ is the adiabatic exponent, equal to $7 / 5$ for a diatomic ideal gas. Noting that the equilibrium volume of the gas in the bubble trap is $S_{b}(H-\bar{x})$, we write

$$
U=\frac{\rho g}{2}\left[S_{b}(\bar{x}+x)^{2}+S_{c}(\bar{y}+y)^{2}\right]+\alpha P_{b, 0} \frac{\left(S_{b}(H-\bar{x})\right)^{\gamma}}{\left(S_{b}(H-\bar{x}-x)\right)^{\gamma-1}}+\alpha P_{a, 0}\left(\frac{V_{a, 0}}{V_{a}}\right)^{\gamma} V_{a} .
$$

Now, we derive an equation of motion from the statement of energy conservation $d E / d t=0$. Differentiating the expressions for $T$ and $U$ with respect to time and eliminating $y$ using $y=-\frac{S_{b}}{S_{c}} x$, we obtain

$$
\begin{aligned}
\frac{d T}{d t} & =\frac{1}{2} \rho\left[S_{b} \dot{x}^{3}+2 S_{b}(\bar{x}+x) \dot{x} \ddot{x}-\frac{S_{b}^{3}}{S_{c}^{2}} \dot{x}^{3}+2 \frac{S_{b}^{2}}{S_{c}}\left(\bar{y}-\frac{S_{b}}{S_{c}} x\right) \dot{x} \ddot{x}+2 \frac{S_{b}^{2}}{S_{l}} L \dot{x} \ddot{x}\right] \\
& =\frac{1}{2} \rho S_{b}\left(1-\frac{S_{b}^{2}}{S_{c}^{2}}\right) \dot{x}^{3}+\rho S_{b}\left[(\bar{x}+x)+\frac{S_{b}}{S_{c}}\left(\bar{y}-\frac{S_{b}}{S_{c}} x\right)+\frac{S_{b}}{S_{l}} L\right] \dot{x} \ddot{x} .
\end{aligned}
$$

The derivative of the adiabatic compression terms is calculated as

$$
\frac{d(\alpha P V)}{d t}=\alpha P_{0} V_{0}^{\gamma} \frac{d}{d t}\left(V^{1-\gamma}\right)=\alpha P_{0} \frac{V_{0}^{\gamma}}{V^{\gamma}}(1-\gamma) \frac{d V}{d t} .
$$

In the limit of an infinite atmosphere (relevant to the conduit side of the system), we find that

$$
\frac{d\left(\alpha P_{a} V_{a}\right)}{d t}=\alpha P_{a, 0}(1-\gamma)\left(-S_{c} \dot{y}\right)=\alpha P_{a, 0}(1-\gamma) S_{b} \dot{x}
$$

The last term in this equation arises because $\frac{d V_{a}}{d t}=S_{b} \dot{x}$, i.e. an increase in the volume of gas in the bubble trap is accommodated by a decrease in the volume of the atmosphere buffering the conduit. Differentiating 
the expression for potential energy $U$ (Equation 2), we find

$$
\begin{array}{r}
\frac{d U}{d t}=\rho g S_{b}(\bar{x}+x) \dot{x}-\rho g S_{b}\left(\bar{y}-\frac{S_{b}}{S_{c}} x\right) \dot{x} \\
-\alpha S_{b} P_{b, 0}(1-\gamma)\left(\frac{H-\bar{x}}{H-\bar{x}-x}\right)^{\gamma} \dot{x}+\alpha S_{b} P_{a, 0}(1-\gamma) \dot{x}
\end{array}
$$

We combine equations 4 and 8 and divide by $\left(\rho S_{b} \dot{x}\right)$ to obtain an equation of motion

$$
\begin{aligned}
{\left[\bar{x}+x+\frac{S_{b}}{S_{c}}\left(\bar{y}-\frac{S_{b}}{S_{c}} x\right)+\frac{S_{b}}{S_{l}} L\right] \ddot{x}=} & -\frac{1}{2}\left(1-\frac{S_{b}^{2}}{S_{c}^{2}}\right) \dot{x}^{2}-g(\bar{x}+x)+g\left(\bar{y}-\frac{S_{b}}{S_{c}} x\right)+ \\
& \alpha \frac{P_{b, 0}}{\rho}(1-\gamma)\left(\frac{H-\bar{x}}{H-\bar{x}-x}\right)^{\gamma}-\alpha \frac{P_{a, 0}}{\rho}(1-\gamma)
\end{aligned}
$$

This nonlinear second order ordinary differential equation can be re-arranged to obtain an expression for $\ddot{x}$ that is suitable for numerical integration. We note that at equilibrium, $\dot{x}=\ddot{x}=x=0$, yielding:

$$
0=-g \bar{x}+g \bar{y}+\alpha(1-\gamma) \frac{P_{b, 0}-P_{a, 0}}{\rho}
$$

because $\alpha(1-\gamma)=-1$, this yields $\rho g(\bar{y}-\bar{x})=P_{b, 0}-P_{a, 0}$ as expected.

\subsection{Oscillation frequency}

In order to find the oscillation frequency of the system described by the equation of motion, we propose that for small oscillation amplitude, the system admits a solution of the form $x=A e^{i \omega t}+B e^{-i \omega t}$ where $A$ and $B$ are (complex) amplitudes. We linearize the adiabatic compression term by truncating a Taylor series expansion, yielding

$$
-\alpha S_{b} P_{b, 0}(1-\gamma)\left(\frac{H-\bar{x}}{H-\bar{x}-x}\right)^{\gamma} \dot{x} \approx-\alpha S_{b} P_{b, 0}(1-\gamma)\left(1+\frac{\gamma x}{H-\bar{x}}\right) \dot{x} .
$$

Substituting the linearization in Equation 11 and expressions for $x$ and its derivatives, we find

$$
\begin{aligned}
{\left[\bar{x}+x+\frac{S_{b}}{S_{c}}\left(\bar{y}-\frac{S_{b}}{S_{c}} x\right)+\frac{S_{b}}{S_{l}} L\right]\left(-\omega^{2} x\right)=- } & \frac{1}{2}\left(1-\frac{S_{b}^{2}}{S_{c}^{2}}\right)\left(-\omega^{2} x^{* 2}\right)-g(\bar{x}+x)+g\left(\bar{y}-\frac{S_{b}}{S_{c}} x\right) \\
& +\alpha \frac{P_{b, 0}}{\rho}(1-\gamma)\left(1+\frac{\gamma x}{H-\bar{x}}\right)-\alpha \frac{P_{a, 0}}{\rho}(1-\gamma),
\end{aligned}
$$

where $x^{*}$ denotes the complex conjugate of $\mathrm{x}$. We now drop all terms of $O\left(x^{2}\right)$ and differentiate once more with respect to time to remove constant terms, yielding

$$
-\left(\bar{x}+\frac{S_{b}}{S_{c}} \bar{y}+\frac{S_{b}}{S_{l}} L\right)(2 \pi \omega)^{2} \dot{x}=-g \dot{x}-g \frac{S_{b}}{S_{c}} \dot{x}+\alpha \frac{P_{b, 0}}{\rho}(1-\gamma) \frac{\gamma \dot{x}}{H-\bar{x}} .
$$

Dividing by $\dot{x}$, we solve for the angular frequency of oscillation

$$
\omega^{2}=\frac{g\left(1+\frac{S_{b}}{S_{c}}\right)+\frac{P_{b, 0}}{\rho} \frac{\gamma}{H-\bar{x}}}{\bar{x}+\frac{S_{b}}{S_{c}} \bar{y}+\frac{S_{b}}{S_{l}} L} .
$$

In the special case of an open system with $S_{b}=S_{c}$, no lateral connector, and no confining pressure in the bubble trap, this expression reduces to $\omega=\sqrt{\frac{g}{\bar{x}}}$, which is the well-known oscillation frequency of fluid in a U-tube (Menneret, 1911, Iguchi et al., 1982). 

diameter by a factor of more than 2 (i.e., going from 1" to $3 / 8$ " diameter) has a small effect (increase) on the oscillation frequency over most of the parameter range that becomes more pronounced at very small values of $\bar{x}$.

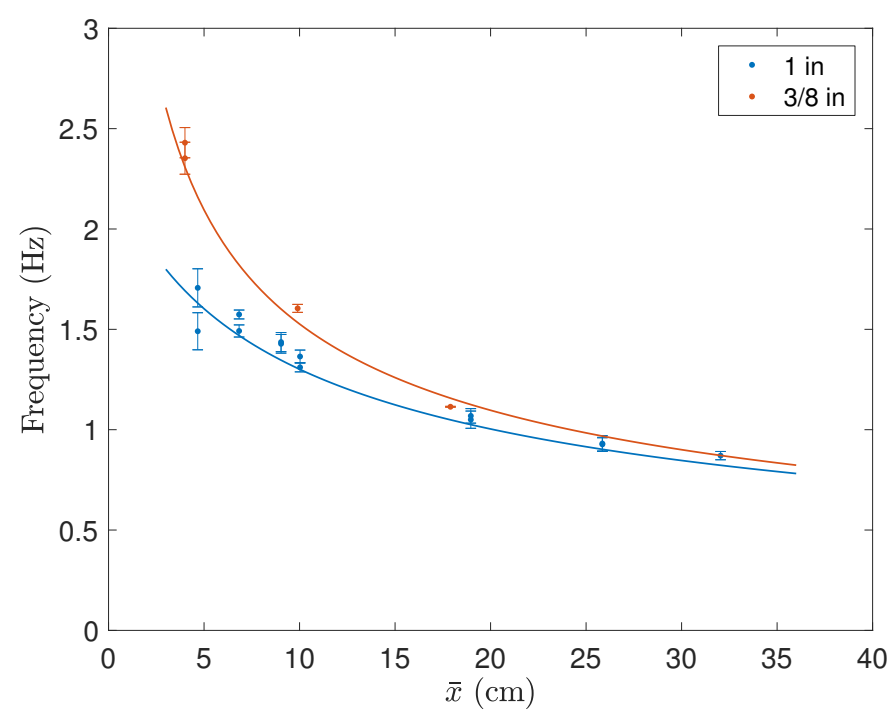

Figure 4: Summary of open-system experiments for 3/8-inch (red) and 1-inch (blue) conduits. Error bars show 95\% confidence interval from fit to either pressure or video time series. The red and blue curves indicate theoretical predictions from Equation 14

The majority of our experiments were conducted with trapped gas in the reservoir, which most closely approximates conditions expected for natural geyser systems. The primary effect of trapped gas is to increase the oscillation frequencies for the largest values of $\bar{x}$, corresponding to small volumes of trapped gas, where gas compression significantly stiffens the oscillating system and increases the resonant frequency (Figure 5). 
This trend is also in good agreement with our theoretical predictions.
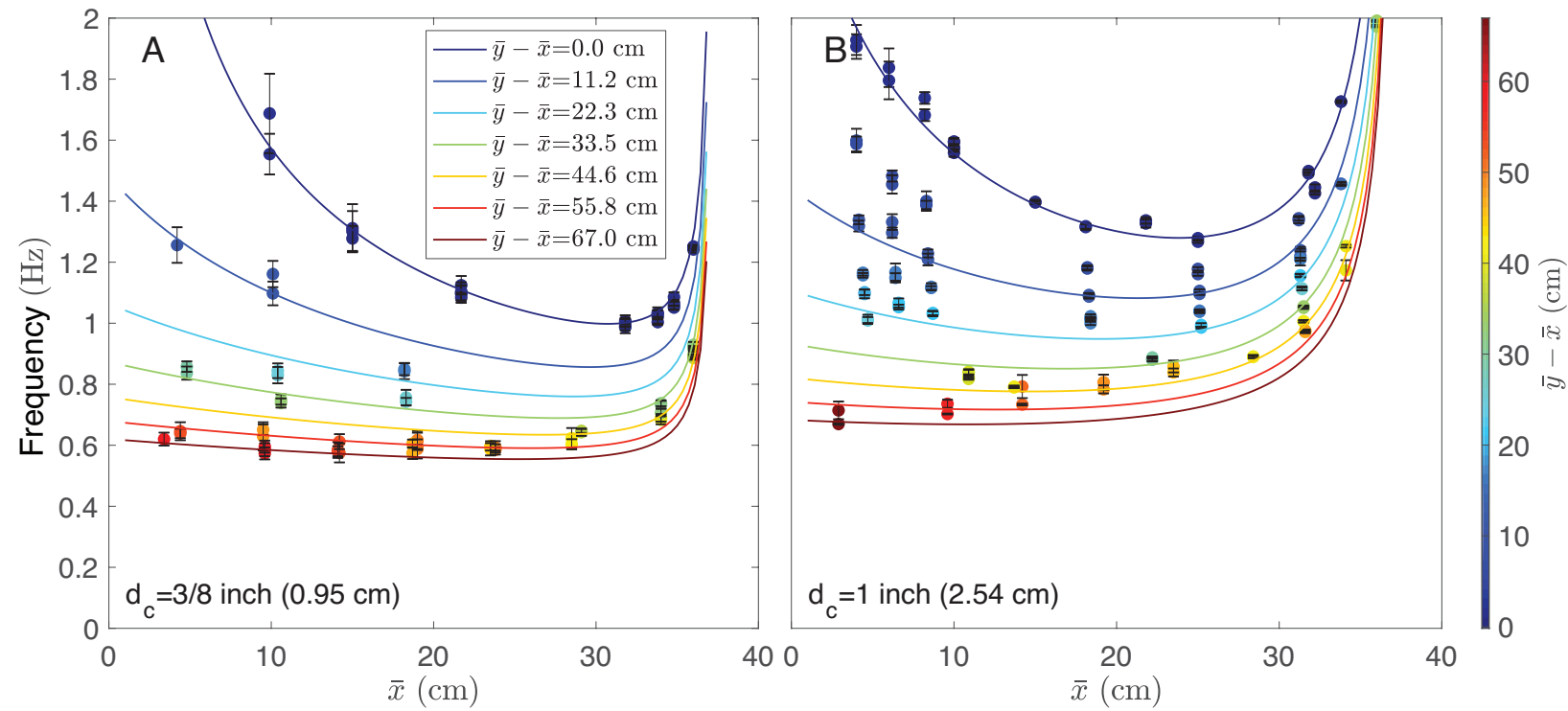

Figure 5: Summary of predicted (Equation 14 colored contours) and observed (colored circles) oscillation frequencies for closed-system experiments. (A) Closed experiments with $d_{c}=3 / 8$ in. (B) Closed experiments with $d_{c}=1$ in.

Overall, our new theory accurately predicts the resonant oscillation frequency for essentially all of our experimental runs (Figure 6). The largest deviations occur at conditions corresponding to very small values of $\bar{x}$ or $H-\bar{x}$, where relative uncertainties of these parameters are largest. 

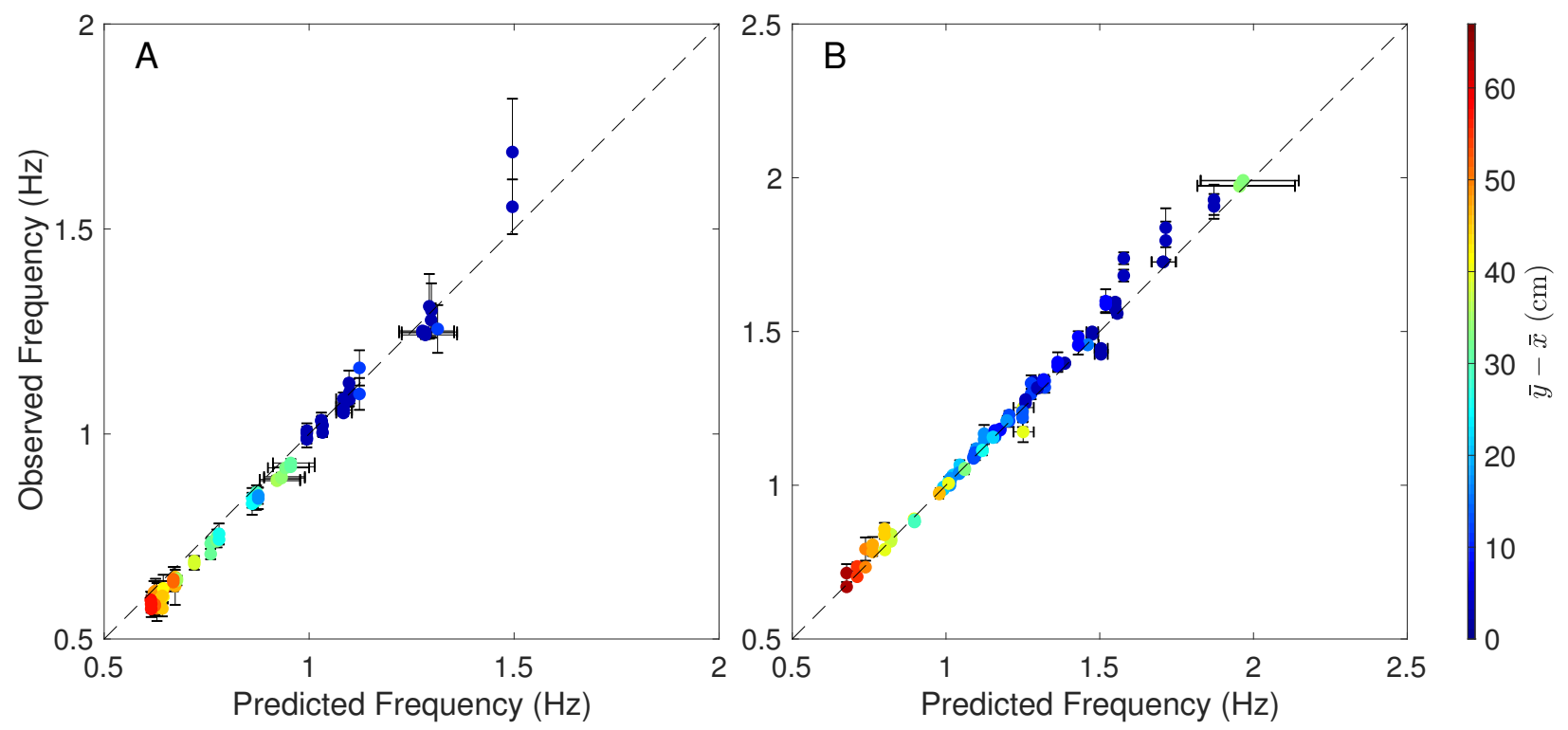

Figure 6: Experimentally determined frequency (vertical axis) and predicted frequency (horizontal axis). The horizontal axis error bars represent $2 \mathrm{~mm}$ uncertainty in determining bubble trap liquid level. Experiments with 3/8-inch and 1-inch conduit are shown in panels A and B, respectively. The diagonal dashed line indicates perfect agreement between prediction and observation.

\section{Discussion}

Our laboratory experiments and theoretical developments are aimed at understanding fluid oscillations in geysers with a bubble trap. Our experimental results demonstrate that the system behaves as a damped oscillator, and our theory accurately predicts the resonant oscillation frequency based on the conduit geometry and the mass of gas and liquid in the system. Our results thus provide a framework for using fluid oscillations to constrain hard-to-observe subsurface parameters, such as the total length of the subsurface plumbing system, in natural systems. In this section we discuss the implications of our results for geyser studies, review the limitations of our theory and experiments, and identify key outstanding issues.

\subsection{Oscillation frequency}

Geophysical measurements have shown that the fluids inside geyser conduit systems oscillate at characteristic frequencies ranging from fractions of a second to minutes Birch and Kennedy, 1972, Hutchinson et al., 1997, Vandemeulebrouck et al., 2014: Karlstrom et al., 2013, Wu et al., 2017), even during recharge periods when the geyser is quiescent, and that the oscillation frequency can change in systematic ways (Rudolph and Sohn, 2017). Developing a framework for modeling and interpreting the frequency of these oscillations is a primary goal of this work. Under controlled laboratory conditions we found that fluids in 
a bubble trap conduit configuration behave as a damped oscillator in response to step-like perturbations to the liquid level, with a deterministic resonant frequency, as expected. However, the resonant frequency did not agree with our previous predictions Rudolph and Sohn (2017), which necessitated a revision of that work. Upon inspection, we found that the Rudolph and Sohn (2017) theory did not correctly account for the force multiplication associated with the difference in conduit and bubble trap cross-sectional areas when $S_{b} \neq S_{c}$. As a result, the equation of motion developed in that work is only valid if the bubble trap and eruption conduit have the same diameter. Reformulating the problem in a Hamiltonian framework simplifies the process of deriving the EOM and reveals similarities with other oscillating systems, most notably the U-tube, which has been used extensively as a model for study oscillatory flows through bent pipes. The pscillation frequency predicted by Equation 14 differs from the corresponding Equation 11 in Rudolph and Sohn $(2017)$ in that the earlier work had $\frac{S_{b}}{S_{c}} \bar{x}+\bar{y}+\frac{S_{L}}{S_{c}} L$ as opposed to $\bar{x}+\frac{S_{b}}{S_{c}} \bar{y}+\frac{S_{b}}{S_{l}} L$ in the present work. For comparison, we show the observed oscillation frequencies and predicted oscillation theories using Equation 11 from Rudolph and Sohn (2017) in Figure S1, which is directly comparable to Figure 5.

We begin by considering the simple case where the conduits are all of uniform diameter and the bubble trap has no lid - i.e., the gas pressure at both liquid interfaces is constant and equal to atmospheric values. This is the classic 'U-tube' physics problem, where a constant diameter tube containing water is bent upwards into a 'U' shape. At equilibrium, the water level at both ends of the tube is equal, and when the water level is perturbed (by blowing into one end of the tube, for example) the system oscillates at a natural frequency determined solely by the length, $L_{U}$, of the water-filled portion of the tube (Menneret, 1911),

$$
\omega=\sqrt{\frac{2 g}{L_{U}}} .
$$

Our equation of motion reduces to this form when the appropriate simplifications are made $\left(L_{U}=\bar{x}+\bar{y}+L\right)$. The most important aspect of this result is that the oscillation frequency does not depend on the conduit diameter nor the details (e.g. radius of curvature) of the conduit path. For all the complexity that the shape and path of a natural geyser conduit system may have, to first order the resonant frequency of the oscillating fluids depends only on the length of the water-filled portion of the conduit and bubble trap.

Next we can consider the impact of trapped gas on the system behavior. If a layer of gas is trapped between the bubble trap liquids and roof, then the gas contributes to the total system potential energy due to energy storage associated with compression. Motion of the liquid in the conduit compresses the gas and thus changes both the gravitational potential energy of the liquid and the internal energy of the gas. This has the effect of stiffening the oscillatory system, and the magnitude of this effect depends on the gas pressure and volume, which are included in our analytical expression for resonant frequency (Equation 14 second term in numerator) by the equilibrium values for the gas pressure, $P_{b, 0}$, and the distance between the bubble trap roof and the gas-liquid interface, $H-\bar{x}$. If the equilibrium gas pressure is high and/or the gas volume is small, then the stiffening effect of the trapped gas is significant and the oscillation frequency increases. 
By contrast, if the equilibrium gas pressure is close to atmospheric and/or the gas volume is large, then the stiffening effect is negligible. Overall, the minimum resonant frequency of the system is determined by the length of the fluid filled portion of the conduit and bubble trap, and gas compression can only increase this frequency.

Finally, we can consider the impact of variable conduit diameters on the oscillation frequency. When considering the 'U-tube' problem we found that the conduit size does not affect the oscillation frequency because it affects the potential and kinetic terms equally and thus is canceled out of the EOM. In our theory for a bubble trap conduit configuration we consider a system with three distinct conduit sizes - one for the bubble trap $\left(S_{b}\right)$, one for the lateral connector $\left(S_{l}\right)$, and one for the eruption conduit $\left(S_{c}\right)$. This formulation allows us to use observations from the laboratory experiments to test our EOM, and it allows us to assess the effect that variable conduit diameters have on the system's oscillation frequency.

Inspection of Figures 5 5 demonstrates that our analytical expression for oscillation frequency (Equation 13) accurately captures the effect of variable conduit diameters. The diameter of the bubble trap and eruption conduit affect the gravitational potential energy term (first term in the numerator), while the diameter of all three components (bubble trap, eruption conduit, and lateral connector) affect the kinetic energy term (denominator). As a result, the conduit diameter effect does not quite cancel out, even for an open system. For an open system (Figure 4 ) the magnitude of the difference depends on the relative contributions from $L$, the length of the lateral connector, and $\bar{x}$ and $S_{b} / S_{c} \bar{y}$, the water level in the bubble trap (or eruption conduit). If $L$ is small relative to $\bar{x}$ and $S_{b} / S_{c} \bar{y}$, the terms nearly cancel each other out and the effect becomes negligible, but if $L$ is similar to, or greater than, $\bar{x}$, then the effect becomes important.

A somewhat different scenario is seen in the closed-system data. The internal energy of the gas does not depend on the conduit diameters except through the relationship between $H-\bar{x}$ and gas volume (i.e., $V_{b}=S_{b}(H-\bar{x})$ ), and therefore this term (second term in numerator of Equation 14) is insensitive to variations in the conduit system diameter. As such, when there is pressurized gas in the bubble trap, the oscillation frequency is less sensitive to variations in conduit size than an open system, and this can be seen by comparing the colored curves for the two different eruption conduit diameters in Figure 6. Over most of the parameter range the two sets of curves are nearly identical, and significant variations are only observed when the bubble trap liquid level is very close to the bubble trap roof (i.e., large values of $\bar{x}$ ), which is an asymptotic part of the solution space corresponding to very large gas compression $\left(\frac{d V}{V}>1\right)$, where the oscillation frequency goes to infinity as $H-\bar{x}$ goes to zero. Because the trapped gas is much more compressible than the liquid in our experiments, we ignored liquid compressibility. If liquid compressibility were included, the oscillation frequency would remain finite.

To summarize, the resonant oscillation frequency of fluids in a geyser with a bubble trap is primarily a function of the length of the liquid-filled portion of the conduit system and the amount and pressure of gas 
in the bubble trap. As the length of the liquid-filled portion of the conduit system increases, the oscillation

frequency decreases, and this term sets the lower limit on the oscillation frequency. Decreasing the amount of trapped gas in the bubble trap leads to increasing oscillation frequency. Variations in conduit diameter exert a weaker influence on the oscillation frequency that can be neglected for most practical purposes except when the fluid mass in the lateral connector becomes significant.

\subsection{Dissipation}

Fluid motion in our laboratory geyser is damped by viscous dissipation. In general, the rate of dissipation depends on a variety of factors, including whether the flow is laminar, turbulent, or transitional, and the detailed conduit geometry. The dissipation in the specific geometry of our laboratory geyser has not been quantified, but dissipation in some relevant systems, including oscillatory flows through straight tubes (Ohmi et al., 1982), flows around right-angle junctions in ductwork, oscillatory flows at the junction between a large reservoir and a pipe (Smith and Swift, 2003), and flows within U-tube manometers consisting of one or multiple loops (Olson and Swift, 1996) has been characterized.

The geometrically simple case of oscillatory flow through a smooth cylindrical pipe is well understood. The structure of flow depends on both the Reynolds number (defined based on the maximum velocity $\langle u>$ ) and also the ratio between the viscous penetration depth and the conduit radius. During oscillations in laminar inertial flow, momentum diffuses across the boundary layer separating the interior of the conduit from its margins. A laminar boundary layer grows with a characteristic length scale of $\delta_{\nu}=\sqrt{2 \nu / \omega}$ where $\nu$ is the kinematic viscosity, which controls the diffusion of momentum across the boundary layer. Because the oscillation of flow introduces an additional timescale to the problem, two dimensionless numbers are required to describe the dynamics of flow. We use first the Reynolds number defined using the maximum velocity $R e=d_{c}\langle u\rangle / \nu$ and second the relative ratio of conduit diameter and the viscous penetration depth $d_{c} / \delta_{\nu}=\left(d_{c} / 2\right) / \sqrt{2 \nu / \omega}$ (e.g. Olson and Swift, 1996). To estimate the conditions under which our experiments were run, we adopt a representative angular frequency $\omega=10 \mathrm{~s}^{-1}, \nu=10^{-6} \mathrm{~m}^{2} / \mathrm{s}$, and a characteristic velocity of $1 \mathrm{~m} / \mathrm{s}$ (based on $10 \mathrm{~cm}$ amplitude oscillations at the representative frequency). The viscous penetration depth is $4.5 \times 10^{-4} \mathrm{~m}$. The Reynolds number is $9.5 \times 10^{3}$ or $2.5 \times 10^{4}$ for the $3 / 8$-inch and 1 -inch conduits. $d_{c} / \delta_{\nu}$ lies in the range of $\sim 10-30$. Under these conditions, flow in the conduit is likely to be laminar or transitional (Ohmi et al. 1982, Olson and Swift, 1996). The center of the conduit will experience plug flow, and a thin laminar boundary layer will develop around the margins of the conduit.

As energy is dissipated from the system, the oscillation amplitude decays towards zero. As shown in Figure 3, the decay of the oscillations is very well approximated by an exponential decay function with the form $(a \cos (\omega t)+c \sin (\omega t)) e^{-t / b}$. The values of the decay timescale $b$ obtained from analysis of the pressure data are shown in Figure 7. The decay timescale is shorter generally for smaller conduit inner diameter and longer for the larger (1-inch) conduit (Figure 7). We also observed an association of shorter decay timescales 
with higher oscillation frequencies.

Energy dissipation can be quantified in terms of a dimensionless quality factor $Q^{-1}=\dot{E} /(\omega E)$. The total energy of the system relative to the equilibrium state can be written

$$
\begin{array}{r}
\Delta E=\frac{1}{2} \rho\left[S_{b}(\bar{x}+x) \dot{x}^{2}+S_{c}(\bar{y}+y) \dot{y}^{2}+S_{L} L\left(\frac{-S b}{S_{L}} \dot{x}\right)^{2}\right]+ \\
\frac{\rho g}{2}\left[S_{b}\left(2 \bar{x} x+x^{2}\right)+S c\left(2 \bar{y} y+y^{2}\right)\right]+ \\
\alpha P_{b, 0} S_{b}\left[\frac{(H-\bar{x})^{\gamma}}{(H-\bar{x}-x)^{\gamma-1}}-(H-\bar{x})\right]+\alpha P_{a, 0}(1-\gamma) S_{b} x .
\end{array}
$$

We calculate the quality factor from the experimental data by numerically differentiating the de-noised pressure time series and converting to equivalent values of $x$ and $y$ (Figure 8). Then we fit an exponential curve to the time series of energy loss to obtain the inverse quality factor. Olson and Swift (1996) derive an approximate analytic expression for the quality factor in oscillatory flow through a smooth, straight pipe and find that $Q \approx Q_{0}-0.75+0.2 / Q_{0}$ with $Q_{0}=d_{c} / \delta_{\nu}$. This equation does not collapse our data onto a single curve, instead producing two curves in $Q-Q_{0}$ space corresponding to the two conduit sizes. We find that our experimental data at lower values of Re can be collapsed onto a single curve for both 1-inch and 3/8-inch conduits if we instead scale the quality factor with $\frac{d_{c}}{\delta_{\nu}} \sqrt{\frac{S_{b}}{S_{c}}}$. The additional factor of the area ratio is physically motivated by the observation that dissipation in pipe flows scales with the ratio of the cross-sectional area over which energy is stored (as fluid inertia) to the area over which energy is dissipated in the laminar boundary layer. We find that our data collected at higher Re do not collapse onto the same curve as the lower Re data, presumably because of a departure from the laminar boundary layer scaling above a critical oscillatory Reynolds number.

\subsection{Relevance to Natural Systems}

In designing the apparatus (Figure 1), our goal was to build a small-scale model of a geyser with a bubble trap that would allow us to study fluid oscillations in the conduit system. We used room-temperature water and noncondensible gas, which is a necessary first step towards experiments that could ultimately utilize hot water and steam. By simplifying the problem in this way, we were able to examine the mechanical behavior of the system at the expense of not including the rich thermodynamic processes that occur in natural geysers (e.g. Lu and Kieffer, 2009). In addition, our laboratory geyser is an idealized configuration with smooth, constant diameter conduits, and as such does not capture all of the flow complexities that are likely associated with the rough and tortuous conduits contained in a natural system.

Our results provide insight into the parameters that control the resonant frequency of fluids in natural geysers with a bubble trap geometry, such as Old Faithful in Yellowstone National Park. While the relevant geometric parameter values for Old Faithful's conduit system are uncertain, we can estimate them for the eruption conduit based on in situ video observations (Hutchinson et al., 1997), and for the bubble trap based 

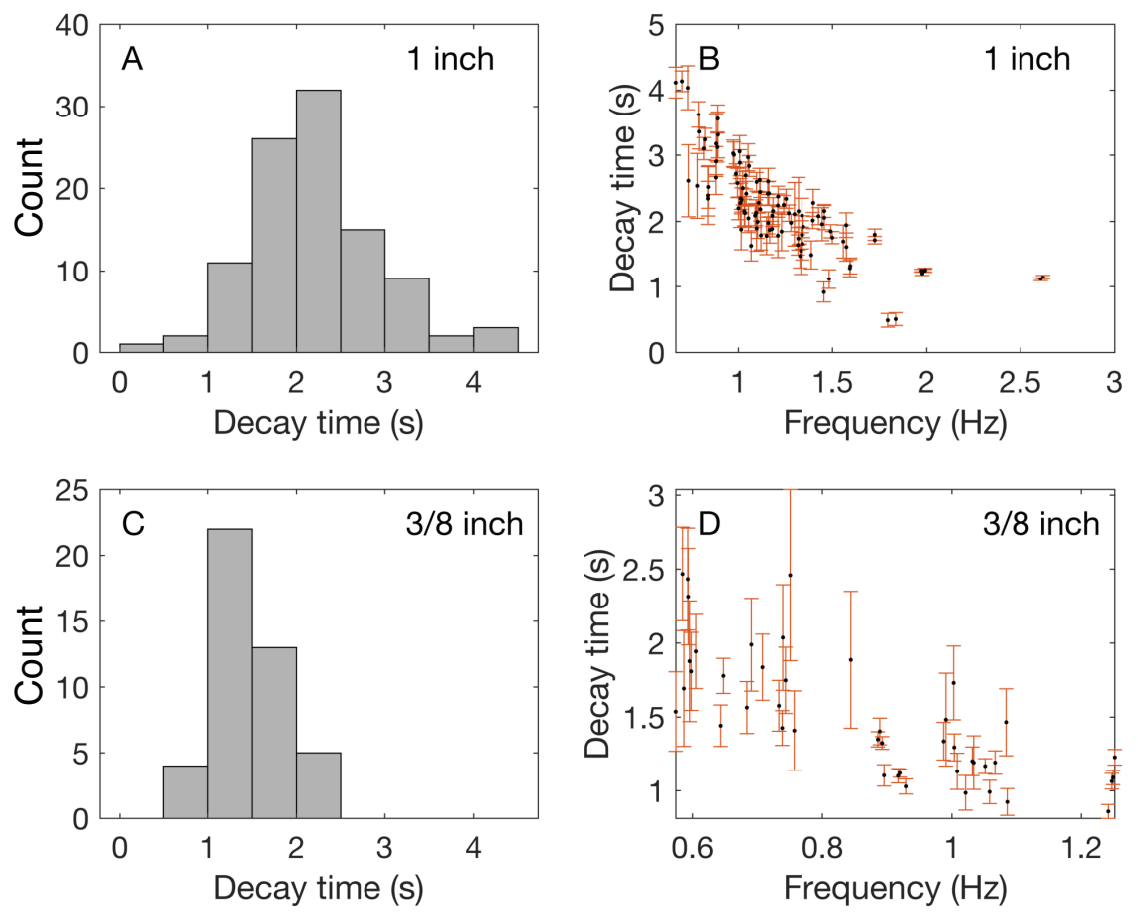

Figure 7: Distributions of oscillation decay timescale $(A, C)$ for all closed-system experiments. In (B,D), we show the decay timescales for individual experiments. Error bars indicate $95 \%$ confidence interval from curve fitting.

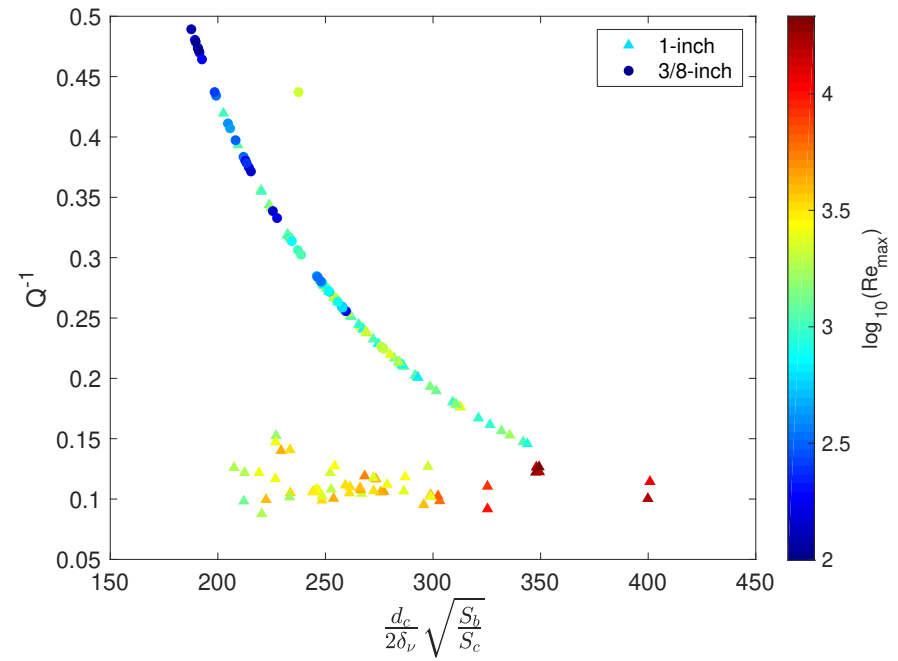

Figure 8: Inverse quality factor $\left(Q^{-1}\right)$ calculated from pressure time series data converted to $x(t)$ and $y(t)$. Triangles and circles indicate values obtained with 1 -inch and 3/8-inch conduits. Color scale indicates maximum oscillatory Reynolds number for each experiment. 
on locations of hydrothermal tremor (Vandemeulebrouck et al., 2013) and Rayleigh wave group velocity measurements (Wu et al. 2017). Using nominal parameter values of $S_{b}=80 \mathrm{~m}^{2}, S_{c}=1 \mathrm{~m}^{2}$, and $H=7 \mathrm{~m}$, we can search the solution space of Equation (14) for combinations of $\bar{y}$ and $H-\bar{x}$ that will yield oscillation frequencies of $\sim 1-1.3 \mathrm{~Hz}$ as observed by pressure gauges in Old Faithful's eruption conduit (Kedar et al., 1998; Rudolph and Sohn, 2017). The minimum resonant frequency corresponding to the limit of gravitational oscillations, $\sim 0.15 \mathrm{~Hz}$ for $L_{U}=22 \mathrm{~m}$ (Equation 15 is much lower than $1 \mathrm{~Hz}$, indicating that the system must be in a state corresponding to the far right hand side of Figure 5 - i.e., a small volume of compressed gas in the reservoir - in order to oscillate at frequencies of $\sim 1 \mathrm{~Hz}$. This is illustrated in Figure 9 which shows the predicted oscillation frequency as a function of the liquid level in the conduit $(\bar{y})$ and the thickness of the gas layer in the bubble trap $(H-\bar{x})$. Interestingly, this part of the parameter space is also where non-linearities in the equation of motion are likely to become most pronounced.

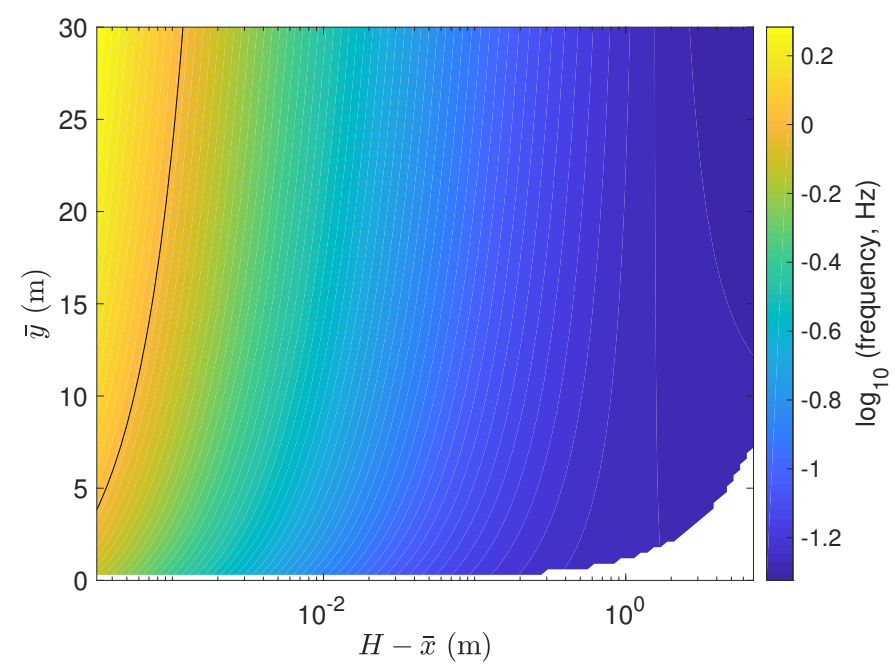

Figure 9: Oscillation frequency $f$ for combinations of $x$ and $y$. Other parameters are $S_{b}=80 \mathrm{~m}^{2}, S_{c}=1 \mathrm{~m}^{2}$, and $H=7 \mathrm{~m}$. The black curve indicates the combination of parameters that yield an oscillation frequency of $1 \mathrm{~Hz}$.

While air was the trapped gas phase used in our experiments and theory, water vapor is likely the dominant trapped gas phase in natural geysers, though noncondensible gas could play a role in some systems (Hurwitz et al. 2016). This difference is important to the oscillations studied here because the compressibility of water vapor may be considerably different from that of an Ideal Gas (Kieffer, 1977), with a corresponding effect on the system's dynamic behavior. In terms of the mathematical model, the effect of water vapor may be implemented by substituting steam table lookups for the adiabatic Ideal Gas equation of state, as was done by Rudolph and Sohn (2017). Conceptually, the greater compressibility of water vapor relative to an Ideal Gas could reduce the stiffening effect of the trapped gas on fluid oscillations, and thereby reduce the impact of the trapped gas on the resonant oscillation frequency. 
The presence of water vapor in the bubble trap also has the potential to give rise to disequilibrium thermodynamic effects that are not incorporated into our theoretical model. Compression of water vapor will change the saturation pressure/temperature of the liquid-gas system, which will lead to mass transfer between the liquid and gas phases as the system fills with fluid in-between eruptions, and potentially also as the system oscillates, depending on the oscillation rate relative to the relaxation time of mass transfer between the two phases as well as the mixing timescale for the water in the bubble trap. However, this mass transfer is beyond the scope of the present work, and is something that needs to be addressed in the future, using laboratory experiments and mathematical models.

Because the conduits of a natural geyser are formed by cavities and fractures in the host rock, they are necessarily much more complicated than those in our laboratory geyser. While we do not yet have good constraints on the shape and size of any bubble traps in natural systems, we do have good constraints on the eruption conduit geometry from a few sites (Hutchinson et al., 1997; Belousov et al., 2013). These studies show that the eruption conduits of some geysers are of nearly constant size and relatively smooth, while others exhibit significant size variations, including narrow constrictions, with rough walls that can include fractures penetrating into the host rock. What impact might these conduit complexities have on fluid motion?

In our apparatus the cross-sectional area of the bubble trap is much larger than that of the eruption conduit, yielding area ratios of 121 and 864 for the 1" and 3/8" diameter conduits, respectively. Although we do not yet have reliable size estimates for the bubble trap in any natural system, it is likely that the area ratios we utilized in our experiments are larger than those of most natural geysers. Given that these large conduit diameter variations exerted only a small effect on the resonant oscillation frequency in our experiments, we expect that the effect will be negligible in natural systems to first-order.

While conduit size variations may not have a significant effect on the system resonant oscillation frequency, they may nevertheless have a large impact on dynamic behavior through the pressure changes they generate when the fluid is moving. Constrictions in the conduit system are effectively nozzles, and the local fluid pressure may drop significantly when flow velocities are high. These pressure drops accelerate the fluid, and if they are large enough they may trigger flashing of hot liquid to steam, fragmenting and further accelerating the flow.

\section{Conclusions}

We performed a suite of laboratory experiments to study oscillations in an analog to natural geysers. The observed oscillation frequencies were not in good agreement with previous theoretical predictions, and we present a revised prediction of oscillation frequencies that are in very good agreement with the laboratory data. Applied to Old Faithful Geyser, we can explain the observed frequency of oscillation for some 
combinations of conduit and reservoir geometry and the gas-to-liquid ratio in the bubble trap. Future work should explore the effect of hot water and steam vs. ideal gas in the bubble trap and quantify the response of the system to controlled inputs of mass and heat, which may excite complex, non-equilibrium processes in the bubble trap and conduit, and investigate the non-linearities in the equation of motion.

\begin{tabular}{|c|l|c|l|}
\hline Symbol & Description & Symbol & Description \\
\hline$d_{c}$ & Conduit diameter & $d_{b}$ & Bubble trap diameter \\
$S_{c}$ & Conduit cross-sectional area & $S_{b}$ & Bubble trap cross-sectional area \\
$S_{l}$ & Lateral connector cross-sectional area & $L$ & Lateral connector length \\
$\bar{x}$ & Equilibrium liquid level in bubble trap & $x$ & Bubble trap liquid level above equilibrium \\
$\bar{y}$ & Equilibrium liquid level in conduit & $y$ & Conduit liquid level above equilibrium \\
$\delta_{x y}$ & Difference between $\bar{y}$ and $\bar{x}$ & $\nu$ & Kinematic viscosity of water \\
$H$ & Height of bubble trap & $L_{U}$ & Total fluid-filled length \\
$P_{a}$ & Atmospheric pressure & $V_{a}$ & Volume of atmosphere \\
$P_{b}$ & Bubble trap pressure & $V_{a}$ & Volume of bubble trap \\
$p_{1}$ & Pressure at transducer 1 Figure 1) & $p_{2}$ & Pressure at transducer 2 \\
$\rho$ & Density of water & $g$ & Gravitational acceleration \\
$\alpha$ & Number of degrees of freedom divided by 2 & $\gamma$ & Adiabatic index \\
$E$ & Total energy & $T$ & Kinetic energy \\
$U$ & Potential energy & $Q$ & Quality factor \\
$\omega$ & Angular frequency of oscillation & $<u>$ & Maximum oscillatory velocity \\
$\delta_{\nu}$ & Viscous penetration depth & \\
\hline
\end{tabular}

Table 2: Summary of mathematical symbols used.

\section{References}

Adelstein, E., Tran, A., Saez, C.M., Shteinberg, A., Manga, M., 2014. Geyser preplay and eruption in a laboratory model with a bubble trap. Journal of Volcanology and Geothermal Research 285, 129-135. doi: $10.1016 / j \cdot j v o l g e o r e s .2014 .08 .005$,

Alexandrov, D.V., Bashkirtseva, I.A., Ryashko, L.B., 2016. Analysis of noise-induced eruptions in a geyser model. The European Physical Journal B 89, 62. doi:10.1140/epjb/e2016-60982-0

Anatolyevich, K.D., 2013. Thermo-mechanical model of geysers. International Journal of Mechanic Systems Engineering 3, 58-66. 
Hurwitz, S., Clor, L.E., McCleskey, R.B., Nordstrom, D.K., Hunt, A.G., Evans, W.C., 2016. Dissolved gases in hydrothermal (phreatic) and geyser eruptions at yellowstone national park, usa. Geology 44, 235-238. doi $10.1130 /$ G37478.1.

Hutchinson, R.A., Westphal, J.A., Kieffer, S.W., 1997. In situ observations of old faithful geyser. Geology 375

Iguchi, M., Ohmi, M., Maegawa, K., 1982. Analysis of free oscillating flow in a U-shaped tube. Bulletin of JSME 25, 1398-1405. doi $10.1299 /$ jsme1958.25.1398

Ingebritsen, S.E., Rojstaczer, S.A., 1996. Geyser periodicity and the response of geysers to deformation. Journal of Geophysical Research 101, 21891-21906. doi 10.1029/96JB02285

380

Kagami, H., 2012. AN EXTENDED DYNAMICAL MODEL OF A GEYSER INDUCED BY INFLOW OF GAS: CONSIDERING EFFECTS OF A COMPLICATED UNDERGROUND WATERCOURSE. World Scientific Publishing Company. pp. 103-111. doi:10.1142/9789812838124_0009.

Karlstrom, L., Hurwitz, S., Sohn, R., Vandemeulebrouck, J., Murphy, F., Rudolph, M.L., Johnston, M.J.S., Manga, M., McCleskey, R.B., 2013. Eruptions at lone star geyser, yellowstone national park, usa: 1. 385 kamchatka, russia, and their geological framework: Implications for the geyser mechanism. Geology 41, 387-390. doi $10.1130 /$ G33366.1.

Birch, F., Kennedy, G., 1972. Notes on geyser temperatures in iceland and yellowstone national park. Geophys. Monog., Am. Geophys. Union 16, 329-333.

Cros, E., Roux, P., Vandemeulebrouck, J., Kedar, S., 2011. Locating hydrothermal acoustic sources at old faithful geyser using matched field processing. Geophysical Journal International 187, 385-393.

Dowden, J., Kapadia, P., Brown, G., Rymer, H., 1991. Dynamics of a geyser eruption. Journal of Geophysical Research 96, 18059-18071. doi 10.1029/91JB01584.

$25,875-878$.

— energetics and eruption dynamics. Journal of Geophysical Research (Solid Earth) 118, 4048-4062. doi 10.

Belousov, A., Belousova, M., Nechayev, A., 2013. Video observations inside conduits of erupting geysers in 1002/jgrb.50251.

Kedar, S., Kanamori, H., Sturtevant, B., 1998. Bubble collapse as the source of tremor at old faithful geyser. Journal of Geophysical Research 103, 24283-24300. doi 10.1029/98JB01824. 
Kedar, S., Sturtevant, B., Kanamori, H., 1996. The origin of harmonic tremor at old faithful geyser. Nature

Kieffer, S.W., 1977. Sound speed in liquid-gas mixtures: water-air and water-steam. J. Geophys. Res. 82, 2895-2904.

Lu, X., Kieffer, S.W., 2009. Thermodynamics and mass transport in multicomponent, multiphase h2o q systems of planetary interest. Annu. Rev. Earth Planet. Sci. 37, 449-477. doi:doi:10.1146/annurev. earth.031208.100109.

Mackenzie, G., 1811. Travels in the Island of Iceland. Allam and Company, Edinburgh.

Menneret, M., 1911. Mouvement oscillatoire et mouvement uniforme des liquides dans les tubes cylindrique. frottement interne. J. Phys. Theor. Appl. 1, 753-766.

Munoz-Saez, C., Manga, M., Hurwitz, S., Rudolph, M.L., Namiki, A., Wang, C.Y., 2015a. Dynamics within geyser conduits, and sensitivity to environmental perturbations: Insights from a periodic geyser in the $\mathrm{El}$ Tatio geyser field, Atacama Desert, Chile. Journal of Volcanology and Geothermal Research 292, 41-55. doi $10.1016 / j \cdot$ jvolgeores.2015.01.002

Munoz-Saez, C., Namiki, A., Manga, M., 2015b. Geyser eruption intervals and interactions: Examples 7 from el tatio, atacama, chile. Journal of Geophysical Research (Solid Earth) , 7490-7507doi:10.1002/ 2015JB012364,

Namiki, A., Munoz Saez, C., Manga, M., 2014. El cobreloa: A geyser with two distinct eruption styles. Journal of Geophysical Research (Solid Earth) 119, 6229-6248. doi:10.1002/2014JB011009.

O'Hara, K.D., Esawi, E.K., 2013. Model for the eruption of the old faithful geyser, yellowstone national park. GSA Today 23, 4-9.

Ohmi, M., Iguchi, M., Kakehashi, K., Masuda, T., 1982. Transition to turbulence and velocity distribution in an oscillating pipe flow. Bulletin of JSME 25, 365-371. doi:10.1299/jsme1958.25.365

Olson, J.R., Swift, G.W., 1996. Energy dissipation in oscillating flow through straight and coiled pipes. The Journal of the Acoustical Society of America 100, 2123-2131. doi:10.1121/1.417922.

Rudolph, M.L., Sohn, R.A., 2017. A model for internal oscillations in geysers, with application to old 415 faithful (yellowstone, usa). Journal of Volcanology and Geothermal Research 343, 17-24. doi:https: //doi.org/10.1016/j.jvolgeores.2017.04.023. 
Smith, B.L., Swift, G.W., 2003. Power dissipation and time-averaged pressure in oscillating flow through a ․ sudden area change. The Journal of the Acoustical Society of America 113, 2455-2463. doi 10.1121/1. 1564022

Steinberg, G., Merzhanov, A., 1981. Geyser process: its theory, modeling, and field experiment. Part 1. Theory of the geyser process. Modern Geology.

Vandemeulebrouck, J., Roux, P., Cros, E., 2013. The plumbing of old faithful geyser revealed by hydrothermal tremor. Geophysical Research Letters 40, 1989-1993. doi:10.1002/grl.50422.

Vandemeulebrouck, J., Sohn, R.A., Rudolph, M.L., Hurwitz, S., Manga, M., Johnston, M.J.S., Soule, S.A., McPhee, D., Glen, J.M.G., Karlstrom, L., Murphy, F., 2014. Eruptions at lone star geyser, yellowstone national park, usa: 2. constraints on subsurface dynamics. Journal of Geophysical Research (Solid Earth) , 2014JB011526-n/adoi:10.1002/2014JB011526.

Wu, S., Ward Kevin, M., Farrell, J., Lin, F., Karplus, M., Smith Robert, B., 2017. Anatomy of old faithful from subsurface seismic imaging of the yellowstone upper geyser basin. Geophysical Research Letters 44, 10,240-10,247. doi:10.1002/2017GL075255. 